\title{
De la haine du mari au sacrifice du patrilignage en contexte coutumier
}

Le cas de la dame de Croisilles (Normandie, XVIII ${ }^{\mathrm{e}}$ siècle)

Hatred of the husband and its impact on patrilineage in eighteenth-century

Norman custom law

Jérôme Luther Viret

\section{OpenEdition}

Journals

\section{Édition électronique}

URL : https://journals.openedition.org/abpo/4129

DOI : $10.4000 / a b p o .4129$

ISBN : 978-2-7535-7720-6

ISSN : 2108-6443

Éditeur

Presses universitaires de Rennes

Édition imprimée

Date de publication : 18 décembre 2018

Pagination : 131-146

ISBN : 978-2-7535-7718-3

ISSN : 0399-0826

Référence électronique

Jérôme Luther Viret, " De la haine du mari au sacrifice du patrilignage en contexte coutumier ", Annales de Bretagne et des Pays de l'Ouest [En ligne], 125-4 | 2018, mis en ligne le 18 décembre 2020, consulté le 02 mars 2022. URL : http://journals.openedition.org/abpo/4129 ; DOI : https://doi.org/ $10.4000 / a b p o .4129$ 


\title{
De la haine du mari au sacrifice du patrilignage en contexte coutumier

\author{
Le cas de la dame de Croisilles \\ (Normandie, $\mathrm{xVIII}{ }^{\mathrm{e}}$ siècle)
}

\author{
Jérôme Luther VIRET \\ Professeur d'histoire moderne, université de Lorraine, CRULH \\ (Centre de recherche universitaire lorrain d'histoire), EA 3945
}

Si l'on entend mettre au jour la conflictuosité familiale sous l'Ancien Régime, il n'est qu'à puiser à pleines mains dans les archives judiciaires et familiales ${ }^{1}$. Celles-ci regorgent en effet de partages inaboutis, de restitutions de dot refusées ou impossibles, de séparations de biens, de heurts conjugaux. Le thème mobilise les historiens depuis longtemps. Parmi les premiers, Maurice Daumas a trouvé dans les factums d'avocats, documents protéiformes situés à mi-chemin entre l'enquête et les mémoires privés, une source précieuse pour l'étude des litiges familiaux ${ }^{2}$. Bien entendu, soigneu-

1. Signalons, parmi quelques publications récentes, deux numéros des Annales de Démographie historique. Crimes familiaux. Tuer, voler, frapper les siens en Europe du XV au XIXe siècle, $\left(\mathrm{n}^{\circ} 2,2015\right)$ et Familles et justices à l'époque moderne. Autorité, pouvoir, conflits, (no 2, 2009). Citons encore le volume d'études réunies par BeLLAVITIS, Anna et Снавот, Isabelle, La Justice des familles. Autour de la transmission des biens, des savoirs et des pouvoirs (Europe, Nouveau Monde, XII -XIX siècles), Rome, EFR, 2011. Pour la noblesse, signalons DESCIMON, Robert et HADDAD, Élie (dir.), Épreuves de noblesse. Les expériences nobiliaires de la haute robe parisienne (XVI'-XVIII siècles), Paris, Les Belles Lettres, 2010.

2. DAumas, Maurice, L'affaire d'Esclans. Les conflits familiaux au XVIII' siècle, Paris, 1988. L'auteur utilise pour cette étude cinq mémoires d'avocat, ainsi que d'autres matériaux, tels que des actes notariés et d'abondants extraits de correspondance. Les factums sont exploités par les historiens du droit, les historiens de la littérature, et les historiens généralistes. On connaît les travaux de Nadine Bérenguier, positionnés entre la littérature et le droit (BÉRENGUIER, Nadine, «L'Infortune des alliances. Contrat, mariage et fiction au dix-huitième siècle ", Studies on Voltaire and the Eighteenth Century, n ${ }^{\circ} 329,1995$, p. 271-417 et de la même, "Fiction dans les archives : adultère et stratégies de défense dans deux mémoires judiciaires ", Studies on Voltaire and the Eighteenth Century, n ${ }^{\circ} 308,1993$, p. 257279). L'intérêt des littéraires pour cette source se justifie par le fait que les plaidoiries se nourrissaient des figures du champ littéraire pour mieux convaincre les juges. LAVOIR, Lise, Factums et mémoires d'avocats aux $17^{e}$ et $18^{e}$ : un regard sur une société (environ 1620-1760), thèse de doctorat, (sous la direction de Jean Meyer), Université Paris IV, 1987. 
sement rédigés par des tiers, avocats, procureurs, notaires ou écrivains publics, les factums étaient composés de façon à provoquer l'adhésion du destinataire. Ils livrent donc une version très partiale des faits. De la même façon, les demandes d'internement administratif, mémoires ou placets adressés au ministre en charge de la Maison du roi ou de la province, offrent des postures stéréotypées, des portraits à charge ou à décharge n'ayant qu'un rapport incertain avec la réalité. La correspondance, les mémoires et rapports conservés par les intendants, écrits à la suite de la demande d'internement, sont à peine plus loquaces. Ils gomment le contexte familial, négligent l'ancrage du conflit dans le passé, n'offrent souvent que des " récits miniatures ". La plupart des dossiers restent impropres à une étude un peu élaborée des mobiles et des arguments avancés par chacune des parties, des étapes et motifs qui ont pu conduire à l'internement d'un parent. Mais l'analyse systématique de différents corpus - généralement, toute la documentation disponible dans le cadre d'une généralité - a permis d'établir une interprétation générale du recours aux lettres de cachet. Les dossiers révèlent une crise de la famille et de l'autorité du chef de famille. Du côté de l'accusé(e), se manifeste une volonté d'émancipation, une quête d'indépendance s'exprimant quelquefois jusqu'à un âge très avancé. Une majorité de correctionnaires âgés de plus de vingt ans n'ont pas réussi à s'établir, souffrent de désœuvrement, rejettent l'autorité du père ou du mari ${ }^{3}$. S'y mêle, du côté des demandeurs, le souci de la préservation de l'honneur. Le désir de ne pas offrir au public le spectacle de la division domestique ou, pire encore, celui de l'impuissance du père ou du mari justifiait ce recours ${ }^{4}$. Au-delà du détenteur immédiat de l'auto-

LEMONNIER-LeSAGE, Virginie, " De la difficile carrière du procureur Jacques Quinsat. De l'intérêt des factums ", dans Droit, histoire et société. Mélanges en l'honneur de Christian Dugas de la Boissonny, Nancy, PUN, 2008, p. 79-92. VIRET, Jérôme Luther, " Le pouvoir dans la famille. Un mémoire judiciaire du Velay en 1787 ", Histoire et sociétés rurales, $\mathrm{n}^{\circ}$ 26, 2006, p. 169-192. Une publication en ligne a réuni plusieurs contributions sur cette source. "Découverte et valorisation d'une source juridique méconnue : le Factum ou mémoire judiciaire". Journée d'études du 7 juin 2012. Textes réunis par Jacqueline VENDRAND-VOYER. La Revue (Centre Michel de L'Hospital. Université d'Auvergne), nº 3, avril 2013. [en ligne] [http://www.droit.u-clermont1.fr/journee-d-etudes-le-factum-ou-memoire-judiciaire.html].

3. JANDEAUX, Jeanne-Marie, Le roi et le déshonneur des familles. Les lettres de cachet pour affaires de famille en Franche-Comté au XVIII siècle, Paris, École des chartes, 2017, p. 225.

4. La littérature sur les lettres de cachet est abondante. Pour ne citer que les travaux les plus récents : STRAYER, Brian Eugene, Lettres de cachet and social control in the Ancien Régime, 1659-1789, N.Y, Peter Lang, 1992. SAVORNIN, Marie-Noël, Les lettres de cachet pour affaires de famille à Paris au XVIII siècle, thèse, EHESS, 2002. CHOPIN-TuFEL, Élise, "Les demandes d'enfermement dans la généralité de Rouen au XVIII ${ }^{e}$ siècle : le récit de vie comme objet d'histoire ", l'Atelier du Centre de recherches historiques [en ligne], 05 | 2009, mis en ligne le 17 septembre 2009, consulté le 8 janvier 2015. [http://acrh.revues.org/1570 : DOI : 10.4000/acrh.1570]. S'agissant de la Normandie, voir QuÉTEL, Claude, "En maison de force au siècle des Lumières ", dans Marginalité, déviance, pauvreté en France, XIveXIX $X^{e}$ siècles, Cahier des Annales de Normandie, 13, Caen, 1981, p. 43-79. WAUTERs, Éric, " Les femmes séquestrées par lettres de cachet dans la généralité de Rouen au XVIII siècle ", Annales de Normandie, $\mathrm{n}^{\circ}$ 2, 2015, p. 55-82. Sur la question de l'enfermement, voir encore : DEMARS-Sion, Véronique, « L'enfermement par forme de correction paternelle dans les pro- 
rité, la condamnation en justice d'un parent ruinait la réputation de tous les proches. La préservation de l'honneur devenait impérieuse lorsque la famille était en difficulté, " menacée de déclin, à la fortune chancelante, fragilisée ou exposée à l'interruption d'un processus d'ascension sociale à peine entamé ${ }^{5}$ ". La Couronne volait donc au secours des lignages les plus exposés au déshonneur.

La présente contribution, venant à la suite d'un premier travail effectué dans le ressort des généralités de Caen et d'Alençon, soulève la question des spécificités régionales ${ }^{6}$. La question de savoir si l'on faisait d'une procédure un emploi différent selon les provinces, en relation avec des cultures familiales différentes et avec des cultures juridiques distinctes, part d'une observation et de la connaissance que nous avons des régimes coutumiers ${ }^{7}$. La présence importante des veuves en Normandie, très importante même, dans les dossiers de l'intendance, et tout spécialement dans ceux de la généralité de Caen, détonne et intrigue. Les 49 veuves dont l'internement fut réclamé au cours du $\mathrm{XVIII}^{\mathrm{e}}$ siècle dans les deux généralités de Caen et d'Alençon, formaient 13,6 \% d'un effectif constitué par 359 demandes d'internement de femmes ${ }^{8} .18$ veuves, parmi ces 49 , nourrissaient des projets de remariage. Le remariage était en Normandie la première cause d'internement des veuves, avant la dissipation (11 cas), le libertinage et les mauvaises fréquentations ( 8 cas), ou bien encore les vols (6 cas). Chose étonnante, on avait trois plus de veuves menacées d'une lettre de cachet en Normandie qu'en Provence, et encore plus du double du taux constaté en Franche-Comté9 !

Ayons à l'esprit qu'il était délicat d'agir contre un très proche parent au moyen d'une procédure aussi extraordinaire et arbitraire que les lettres de cachet! Compte tenu des sensibilités fortement divergentes des populations du nord et du midi de la France, les unes coutumières, les autres " romaines ", relativement à la nature et à l'étendue du pouvoir domes-

vinces du Nord au XVIII ${ }^{\mathrm{e}}$ siècle ", Revue Historique de Droit Français et Étranger, 3, 2000, p. $429-472$.

5. JANDEAUX, Jeanne-Marie, Le roi et le déshonneur des familles..., op. cit., p. 219.

6. VIRET, Jérôme Luther, "L'internement des veuves et des célibataires majeures. Une exception normande dans la $2^{\mathrm{e}}$ moitié du XVIII ${ }^{\mathrm{e}}$ siècle ", Revista de historiografía, $\mathrm{n}^{\circ} 1,2017$, p. 107-124.

7. S'agissant de la Normandie, je me permets de renvoyer à mon travail : VIRET, Jérôme Luther, La famille normande. Mobilité et frustrations sociales au siècle des Lumières, Rennes, PUR, 2013.

8. Dans le détail, cela représente 187 dossiers féminins pour la généralité de Caen (pour un total de 1723 dossiers de l'un et l'autre sexe) et 172 dossiers pour la généralité d'Alençon (pour un total de 700). Les totaux ont été calculés par QuÉTEL, Claude, De par le Roy..., op. cit., p. 131.

9. EMmANUELLI, François-Xavier, "Ordres du roi et lettres de cachet en Provence à la fin de l'Ancien Régime. Contribution à l'histoire du climat social et politique ", Revue historique, $\mathrm{n}^{\circ} 512$, 1974, p. 375. Le pourcentage de veuves s'élève à 4,5\%. En Franche-Comté, six veuves ont été poursuivies, soit $6,2 \%$ des personnes incriminées de sexe féminin (JANDEAuX, Jeanne-Marie, Le Roi et le déshonneur..., op. cit., p. 221 et 267). 
tique, une différenciation géographique du recours aux internements administratifs paraît plausible. Certains parents en Normandie estimaient que les intérêts du lignage - du patrilignage - devaient prévaloir à l'encontre de femmes libres et capables, qu'elles fussent célibataires ou veuves ${ }^{10}$ ! L'enfermement d'une veuve, pour des motifs autres que l'aliénation ou le vol, exceptionnel hors de Normandie, était ici justifié par les intérêts du lignage masculin. Les mères ne manquaient jamais de souligner l'infamie d'une telle action, surtout lorsqu'elle était engagée par un fils. Au commencement de l'histoire de la dame de Croisilles, un mariage d'intérêt, une union mal assortie en âges et en caractères, conduit à un adultère et à un premier enfermement de l'épouse, suivi de sa libération trois années après. Rien là que de très ordinaire. L'extraordinaire survient lorsque le fils, 27 ans plus tard, réclame contre elle un second enfermement! C'est au caractère implacable du patrilignage normand - implacable en raison de son évidence plutôt que de sa rigueur - ainsi qu'au caractère exceptionnellement bien trempé de la personne enfermée, qu'il faut alors attribuer le recours aux ordres du Roi. La présente étude de cas met en évidence le fait qu'une procédure offerte aux familles, mise au service de "l'intérêt supérieur du lignage ", devait nécessairement conduire au châtiment des éléments les plus opposés à l'idée même du lignage, fût-ce même une veuve âgée de 66 ans. Difficilement contestée en Normandie, l'évidence lignagère s'affaiblissait au fur et à mesure que l'on se rapprochait de Paris et Versailles. L'emportaient alors les droits de la personne majeure. C'est ce que révèle la présente affaire.

\section{Les dérèglements d'une veuve}

L'action se situe à Villers-Bocage, un bourg où le sieur Bernardin de Croisilles tenait le second rang, après le comte Pierre Marie Maximilien Le Vicomte de Blangy ${ }^{11}$. À sa mort, en octobre 1770, la dame de Croisilles, épouse séparée de biens et de corps du sieur Bernardin depuis 23 ans, revient au domicile conjugal. Aux origines de cette séparation d'une épouse d'avec son mari et d'une mère d'avec son fils, puisque de cette union naquit un fils, se trouvent une relation adultère de la femme avec un commis aux aides et l'enfermement dans un couvent obtenu par le mari au moyen d'une première lettre de cachet $^{12}$. On sait peu de chose des 23 années qui sui-

10. Nous qualifions ici de patrilignage les groupes de descendants qui se réclament d'un même ancêtre en ligne agnatique (exclusivement masculine).

11. Né le $1^{\text {er }}$ septembre 1718; marié le 3 août 1753 avec Marie-Anne Françoise Rose Le Berseur; décédé le 24 août 1789. Capitaine dans le régiment d'Ermenonville, exempt des Gardes du corps du Roi, maître de camp de cavalerie, brigadier de Sa Majesté. VillersBocage se situe dans l'actuel département du Calvados, arrondissement de Vire, à $25 \mathrm{~km}$ au sud-ouest de Caen.

12. Arch. dép. du Calvados, C 337, lettre de Saint-Florentin à M. de la Briffe, intendant de Caen, du 15 décembre 1747. Cette lettre fait état de la lettre de cachet délivrée quelques jours plus tôt pour l'enfermement de la dame, d'abord au couvent des bénédictines de Domfront. Des lettres postérieures indiquent qu'elle fut transférée à l'abbaye 
virent l'ordre royal, sinon qu'elle resta enfermée trois années et résida vers la fin pendant 8 à 10 années chez un perruquier de Rennes où elle se prit d'affection pour un enfant, au point de le tenir pour son enfant " adoptif ". En 1778, la dame de Croisilles hérita de la seigneurie de Cardonville, proche de Bayeux. C'était un bien considérable, qui devait revenir plus tard à son fils légitime, François Jean-Charles de Croisilles ${ }^{13}$. C'est à ce moment précisément que les choses dérapent.

À la demande de son fils, une enquête est lancée contre la dame de Croisilles. Plusieurs habitants de Villers-Bocage déposent et relatent les écarts dont cette femme désormais âgée, infirme et sourde, est supposée s'être rendue coupable depuis plusieurs années. Un premier témoin, cordonnier, s'étend sur la présence dans la maison de la dame de Croisilles d'une femme Richebourg, d'une demoiselle Lamare sa cousine, et encore d'un frère de cette dernière " ci-devant soldat ". D'après le témoin, ce soldat se faisait gloire de pouvoir vivre dans la maison aux dépens de la dame. Elle " ne voyait chez elle que ces sortes de gens et autres personnes du bourg qui avaient besoin de sa table et qui prenaient plaisir à entretenir l'éloignement où elle était pour son fils, qu'elle paraissait haïr ${ }^{14}$ ". Parlant des mêmes personnes, un second témoin ajoute que la maîtresse de maison et ses invités passaient " des journées entières à jouer et à se divertir ». Le cordonnier situe les faits sept à huit années plus tôt, c'est-à-dire à l'époque où la dame est revenue au domicile conjugal, tandis qu'un autre témoin, menuisier de son état, ayant effectué des travaux dans la maison pendant trois mois, ayant donc eu tout loisir d'observer ce qui s'y passait, parle de six années. Si la chronologie est quelque peu flottante, la description converge. La dame, déclare le menuisier, "tenait table ouverte ". Les invités s'y succédaient " les uns aux autres, après y avoir demeuré tantôt huit jours, tantôt plus tantôt moins ". Le même témoin se fait plus précis sur le rôle tenu par la nommée Richebourg, officieusement couturière, en réalité plutôt " maîtresse de la maison ". La demoiselle Lamarre, également couturière de son état, "tenait la bourse et payait le déposant ". Ce sont ces personnes qui mirent selon lui dans la tête de la dame de Croisilles l'idée d'acquérir un carrosse à quatre chevaux alors qu'elle avait déjà un

Blanche, bénédictine, proche de Mortain en 1748 puis en la communauté de Montbareil, à Guingamp, en 1749. Elle en est sortie en 1750 .

13. En Normandie, les femmes, nobles ou roturières, n'héritaient qu'en l'absence de garçons. Sur les droits des filles et plus précisément des veuves en Normandie et l'esprit du droit coutumier normand, les publications de Jean Yver apportent de précieux éclairages (YvER, Jean, "Les caractères originaux du groupe de coutumes de l'Ouest de la France ", Revue historique de Droit français et étranger, 1952, nº 1, p. 18-79). Lire également LEMONNIER-LESAGE, Virginie, Le statut de la femme mariée dans la Normandie coutumière. Droit et pratiques dans la généralité de Rouen, Presses Universitaires de la Faculté de droit de Clermont-Ferrand, LGDJ, Clermont-Ferrand, 2005.

14. Arch. dép. du Calvados, C 337, déposition de Michel Delarue, 33 ans, cordonnier à Villers-Bocage, 18 septembre 1778. 
cabriolet $^{15}$. Un témoin, moins fiable, car de son propre aveu ayant servi le fils quelque temps, confirme que la dame de Croisilles avait tout à fait abandonné l'administration de sa maison aux dame et demoiselle Richebourg et Lamarre. L'affaire se conclut devant le bailli de Villers-Bocage par la nomination d'un curateur. Cependant, l'affaire n'en resta pas là.

L'interdiction pour dissipation, soit qu'elle fût accordée à titre temporaire, soit qu'elle n'ait pas totalement privé la dame de sa capacité de disposer, laisse le fils dans une vive anxiété. Il craint en effet qu'elle ne poursuive ses dissipations. Aussi conçoit-il l'idée de la faire fléchir et de lui faire signer une renonciation à aliéner son bien en usant d'un internement par lettre de cachet. L'enfermement de la mère doit se faire discrètement et par surprise. L'intendant, convaincu des intentions de la mère, demande en effet au ministre de ne rien ébruiter des intentions du fils car, affirme-t-il : " Du moment que madame de Croisilles la mère sera instruite des démarches de son fils, elle vendra tout moitié au-dessous de sa valeur ". D'où vient cette forte conviction? Probablement est-elle due à la présence de cet enfant, élevé et comme adopté par la dame depuis l'âge de deux ans. À cet enfant, elle fait sans hésitation donation d'une rente de 200 livres. Son fils légitime est prié par elle de l'appeler "mon frère "! Après avoir de la sorte humilié son fils, elle offre sans raison apparente à son cocher une rente viagère de 100 livres, puis fait la dépense d'un carrosse et de quatre chevaux pour la promenade de ses dames de compagnie. En 1778, la dame de Croisilles, alors âgée de 66 ans, est enfermée à la demande de son fils. Cependant, la manœuvre échoue. La dame refuse de signer quelque écrit que ce soit. Elle connaît son droit, adresse un mémoire au ministre, réclame sa libération :

\footnotetext{
"Excusez, je vous supplie, le refus que je fais de m'engager à garder ma succession aux enfants de Monsieur de Croisilles. J'ai pour cet acte une répugnance naturelle que je ne peux vaincre, mais soyez persuadé Monseigneur que je n'ai aucune haine dans le cœur contre mon fils [...] que je n'ai jamais eu l'intention de le déshériter. Je ne lui ferai aucun tort. J'ai l'honneur de vous en donner ma parole, mais point de signature, ne m'en voulez point de mal $n$ !
}

On voit avec quelle assurance et quelle liberté une veuve résiste aux pressions multiples exercées sur elle. Dans cette attitude, l'affection portée au fils " adoptif " joue certainement un rôle. Mais il y a aussi dans le ton, ironique, et sur le fond, un défi. Il y a le rejet brutal, définitif, d'un mari et de sa descendance, la volonté affichée de s'affranchir d'une logique patrilignagère, particulièrement solide en Normandie. La séparation initiale, déclare-t-elle, n'était pas de son fait. "Comme il [son mari] était fort attaché à son père et à sa mère. Il a toujours voulu demeurer avec eux. Ce que j'ai patienté pendant nombre d'années, mais à la fin, ne pouvant plus y tenir... j'ai pris le parti de me retirer à l'hôtel-Dieu ". Patrilignagère, la Normandie est également, comme toute la France du nord, pays de famille nucléaire et

15. Arch. dép. du Calvados, C 337, déposition de Jean-Baptiste Picard, 30 ans, menuisier à Villers-Bocage, 18 septembre 1778. 
d'établissement néolocal. Le rejet du patrilignage - et de la notion même de lignage - se double d'une défense audacieuse de sa servante, la demoiselle Lamare. "L'obscurité ne dégrade point, surtout quand on la dissipe par une conduite irréprochable et une noblesse de sentiments, bien préférable à celle qu'on reçoit de ses aïeux ${ }^{16}$. " L'argument, banal dans la bouche d'un roturier, est peu commun dans celle d'une femme noble. Impuissant, exposé au déshonneur, le fils se résout à « fermer les yeux sur le danger de la spoliation " et demande la libération de sa mère.

\section{Mon fils n'en veut qu'à ma fortune}

Cette veuve a-t-elle réellement voulu priver son fils d'héritage? Plusieurs témoins l'affirment, qui déclarent l'avoir entendue dire à maintes reprises qu'elle se proposait de vendre son bien pour en frustrer son fils, par haine de celui-ci. Un tel comportement est plausible. La veuve Croisilles reconnaît avoir menacé son fils de " déshéritement ", ajoutant toutefois que c'était uniquement pour le faire rentrer dans son devoir ${ }^{17}$. Enfermée à l'abbaye Blanche de Mortain, elle déclare ne vouloir aucun mal à ses enfants, n'avoir aucune haine dans le cœur, malgré les procédés si terribles employés par son fils. Lyrique, elle conclut : "Je ne pourrais peindre la douleur et l'angoisse dont mon cœur est déchiré au souvenir de la main qui me poi$\operatorname{gnarde}^{18}$ ".

Si l'accusation est vraisemblable, c'est aussi que plusieurs notables habitants et le curé de Villers-Bocage lui-même ont signé une déclaration affirmant qu'elle comblait de bienfaits cet enfant adoptif, ce Gapi dont il a déjà été question, fils d'un perruquier de Rennes, pour le bien-être duquel ladite dame de Croisilles aurait aliéné une bonne partie de sa fortune afin d'en priver son héritier légitime. C'est bien d'un crime patrilignager qu'il s'agit. La dame est obligée de se justifier. Elle explique au ministre, Bertin, qu'elle nourrit cet enfant depuis l'âge de deux ans, qu'il est le fils d'un honnête bourgeois de Rennes, " homme peu fortuné chargé d'une nombreuse famille et auquel [elle doit] des obligations ${ }^{19}$ ". Faut-il croire la déposition du menuisier disant qu'elle a installé Gapi, ce garçon arrivé chez elle à Villers-Bocage vers l'âge de sept ans, dans un lit placé au pied du sien, dans la même chambre? Quelles dépenses a-t-elle véritablement faites pour lui? D'abord, elle a fait venir un prêtre en pension pour qu'il s'occupe de son éducation. Tous les témoins savent ensuite qu'elle a fait donation à Gapi d'une rente de 200 livres en décembre 1770. L'acte établi devant le notaire de Villers-Bocage au profit du bourgeois de Rennes Guillaume

16. Arch. dép. du Calvados, C 337, mémoire de la dame de Croisilles adressé à l'intendant, sd.

17. Arch. dép. du Calvados, C 337, lettre de la dame de Croisilles à Bertin, 2 décembre 1778 .

18. Idem.

19. Arch. dép. du Calvados, C 337, lettre de la dame de Croisilles à Bertin, 11 septembre 1778 et mémoire non daté. 
Agapit - patronyme ayant inspiré le diminutif Gapi - contient une clause importante. Il est spécifié que la rente ne pourra pas aller en ligne collatérale, et qu'elle devra rester en ligne directe au profit uniquement des enfants à naître dudit mineur ${ }^{20}$. Si elle avait uniquement souhaité ruiner son fils, la dame de Croisilles n'aurait pas mis cette clause. Plusieurs témoins l'accusent encore d'avoir fait donation d'une rente de 100 livres à son cocher, Charles Allais; mais il s'agit d'une rente viagère devant s'éteindre à son décès ${ }^{21}$. "Voilà tous les dons que j'ai fait ", conclut-elle. Il n'y a là rien de très extraordinaire. Il est vrai que d'autres sommes peuvent avoir été distraites de diverses façons. La dame de Croisilles a vendu un herbage situé à Cardonville valant 15000 livres, pour 7000 livres payées comptant et le reste faisant une rente de 350 livres. Elle justifie cette vente par un procès intenté à un seigneur voisin, relatif au droit de patronage de la terre de Cardonville ${ }^{22}$. Le surplus a servi à amortir une rente et à faire des réparations sur des chemins. Bref, sans surprise, elle assure avoir correctement géré son bien et avoir porté les loyers de ses terres plus haut que ne l'avait fait son mari. Cette mise au point permet de connaître le revenu de la dame à la date de son second internement : il était de 7400 livres.

La dame de Croisilles se défend d'avoir jamais été avare de son argent et surtout déclare avoir toujours agi dans l'intérêt de son fils! Elle abandonne, à la mort de son mari, douaire et reprises qu'elle avait sur les biens de son époux. La mesure était généreuse. Son mari avait à ses dires aliéné pour 700 livres de ses rentes dont elle aurait pu réclamer les arrérages. Loin de réclamer, elle abandonne ses intérêts pour l'honneur de la mémoire de son mari. Elle agit de même envers son fils et renonce au douaire. "J'en usai en mère tendre envers mon fils et lui abandonnai tous les meubles de la succession, à la réserve de quelque argenterie que je partageai avec lui. Je renonçai à exercer aucun droit de douaire... et ne me réservai qu'un piedà-terre dans la maison de Caen ". Il se peut qu'elle ait alors sincèrement voulu faire la paix, esquisser peut-être un rapprochement avec ce fils qui était pourtant pire qu'un étranger, la continuation du lignage honni. C'est à ce moment qu'elle lui offrit, ainsi qu'à sa récente épouse, de venir dans sa maison, prise à loyer à Villers-Bocage. On ne saura jamais dans quel état d'esprit elle était alors, mais il se peut qu'elle ait voulu solder symboliquement et réellement les comptes avec le lignage de son défunt mari. Sincère ou non, la dame de Croisilles qualifiera rétrospectivement l'engagement de son fils comme " un mariage de fantaisie contracté avec la fille d'un Canadien retiré à La Rochelle ${ }^{23}$ "! La cohabitation cessa au bout de 18 mois

20. Arch. dép. du Calvados, C 337, copie de l'acte établi devant Jean-Baptiste Robert Langlois, notaire à Villers-Bocage, du 20 décembre 1770.

21. Arch. dép. du Calvados, C 337, copie de l'acte établi devant Jean-Jacques Benard, notaire à Caen, le 11 novembre 1774 .

22. Arch. dép. du Calvados, C 337, lettre de la dame de Croisilles à l'intendant, 30 septembre 1778. Le dossier contient une copie de l'acte de vente daté du 6 mars 1778.

23. Arch. dép. du Calvados, C 337, mémoire non daté rédigé par un tiers depuis l'abbaye Blanche de Mortain. 
par la faute, dit-elle, de son fils. " Je les ai logés et nourris gratuitement tant que leur humeur altière ne les [avait] pas portés à me rendre toute habitation insupportable avec eux ${ }^{24}$ ".

Revenons à la question principale, cause de toute la procédure, soit la prétendue dissipation de biens. "À faux ", se plaint-elle, "me donnet-on le titre de dissipatrice ${ }^{25}$ ". Loin d'avoir elle-même porté atteinte à la fortune familiale, elle accuse plutôt son fils d'avoir dépensé et aliéné sans raison. Elle dresse la liste des pertes successives. François Jean-Charles de Croisilles a vendu une " rente de fieffe" pour un capital de 23000 livres et sa terre de La Vaugueux pour 17200 livres, soit un total de plus de 40200 livres, somme grossie peut-être encore par d'autres petites ventes puisque la dame de Croisilles évoque finalement 45000 livres de ventes en moins de six années ${ }^{26}$. Le fils reproche à sa mère de le laisser dans la misère. La vérité est que les revenus de la dame sont plus élevés que ceux du fils. C'est bien là le nœud de toute l'affaire. Pour empêcher la perte du patrimoine familial, il faut que le fils obtienne en justice une sentence d'interdiction. L'interdiction d'une mère n'allait cependant pas de soi! Plusieurs tribunaux observaient encore avec rigueur au XVIII ${ }^{\mathrm{e}}$ siècle l'article 182 de l'ordonnance de Blois (1579) disposant que les veuves qui « même ayans enfans d'autres mariages, se remarient follement à personnes indignes de leur qualité, et qui pis est, les aucunes à leurs valets ", seront interdites. Un arrêt du parlement de Bretagne rendu en 1614 avait même décidé qu'une femme faisant semblable mariage était interdite de plein droit sans aucun jugement ${ }^{27}$. Mais dans le cas qui nous préoccupe, un projet matrimonial était invraisemblable, et l'interdiction pratiquement impossible à obtenir sur un tel soupçon. Les dépenses elles-mêmes, dans le cas de la dame de Croisilles, n'y suffisaient pas. Il n'était pas d'usage d'être déclaré prodigue pour la seule dilapidation des meubles. Pour obtenir l'interdiction, il fallait prouver qu'au moins le tiers des fonds avait été aliéné. Or, la dame de Croisilles n'avait pas vendu en capital le dixième de son bien. C'est l'impossibilité d'obtenir une interdiction définitive qui amène le fils à requérir une lettre de cachet. Le jeune François Jean-Charles de Croisilles trouve des soutiens. Le comte de Blangy, premier seigneur du lieu, expose dans une lettre qu'il n'y a rien de scandaleux dans le recours aux ordres du roi. " On ne fait aucun tort à cette mauvaise mère, on lui conserve sa fortune et on l'empêche de commettre une injustice criante ${ }^{28}$ ". La dame ne veut pas renoncer à sa liberté d'aliéner

24. Arch. dép. du Calvados, C 337, mémoire de la dame de Croisilles remis à Bertin, le 11 septembre 1778 .

25. Arch. dép. du Calvados, C 337, mémoire de la dame de Croisilles à $\mathrm{M}^{\mathrm{r}}$ de Paumier, 7 octobre 1778 .

26. Arch. dép. du Calvados, C 337, mémoire sans date adressé à l'intendant.

27. ClÈRE, Jean-Jacques, "Essai sur l'évolution de l'interdiction en droit français (XVII $\mathrm{xx}^{\mathrm{e}}$ siècles) ", Mémoires de la société pour l'histoire du droit et des institutions des anciens pays bourguignons, comtois et romands, tome 58, 2001, p. 152.

28. Arch. dép. du Calvados, C 337, lettre du comte de Blangy du $1^{\text {er }}$ août 1778. Le destinataire est inconnu mais elle figure dans les papiers de l'intendant. 
et résiste à toutes les pressions. Des négociations sont alors engagées qui ne débouchent sur rien. Le fils remet au ministre un placet en vue d'obtenir la libération de sa mère. Une lettre de celui qui fut un moment le curateur de la dame conclut : " Nous perdons tout le fruit de nos espérances ${ }^{29}$ ".

\section{Mobiliser parents et notables}

Si nous sommes parvenus au dénouement de cette affaire, nous n'avons pas encore saisi tous les enjeux ni souligné suffisamment la singularité de l'affaire. Il faut pour cela apprécier à sa juste valeur l'incroyable brutalité du procédé et le caractère inaccoutumé de l'enfermement d'une mère par son fils, même dans une région comme la Normandie! Si une telle chose était possible, c'est qu'en réalité le fils n'était pas seul à agir. Fut-il même l'initiateur de cet enfermement? La chose n'est pas certaine. L'examen du dossier, dont chaque pièce a été rédigée dans le dessein de convaincre soit l'intendant soit le ministre, ne donne jamais accès qu'à une fiction! Sans appartenir au genre élaboré du factum ou de la plaidoirie imprimée, le placet, les mémoires et rapports qui accompagnaient la demande de lettre de cachet, adoptaient des formes ou figures de style empruntées au champ littéraire, mettaient en scène des faits et des personnages avec un art qui tenait autant, sinon plus, du théâtre que de l'enquête judiciaire. Dans cette tragi-comédie, le rôle principal revient bien entendu à la dame de Croisilles et à son fils. Cependant, d'autres personnages s'agitent en coulisse.

L'affaire éclate devant la justice ordinaire, en l'auditoire de VillersBocage, avec la déposition de plusieurs témoins, à la réquisition du fils ${ }^{30}$. On délibère de l'état de la mère, détenue à l'abbaye Blanche de Mortain, dans le but d'obtenir sa mise sous curatelle. Le bailli Pierre de la Fontaines, après avoir pris l'avis des parents et écouté les témoins, établit le sieur Cauvin de Beaumont comme curateur. Barnabé Jean-Baptiste Charles Cauvin de Beaumont figure en tête de la liste des parents situés du côté paternel. Cauvin de Beaumont est un parent collatéral du requérant puisque l'un et l'autre sont parents par leur grand-mère du côté paternel. En qualité de curateur, il apparaît très résolument en faveur du maintien en détention de la dame de Croisilles. Les autres témoins requis par le fils sont nobles et parents à un degré assez éloigné, comme Jacques Henry Siméon de Croisilles ou bien Jean-Louis d'Arclais de Monbosq, tuteur des enfants de Monsieur de Croisilles de la Mousse ${ }^{31}$. Les parents de la dame de Croisilles, née Marie-Madeleine de Méhérenc, du côté maternel donc, sont également des collatéraux éloignés. Cette absence des parents proches de l'un et l'autre côté s'explique par des pratiques restrictives en matière de fécondité et par des décès précoces. La branche cousine issue du trisaïeul de

29. Arch. dép. du Calvados, C 337, lettre de Cauvin de Beaumont du $1^{\mathrm{er}}$ mars 1779.

30. Arch. dép. du Calvados, C 337, délibération du 9 septembre 1778.

31. Il s'agit de Jacques Philippe de Croisilles de la Mousse décédé en 1769 à l'âge de

40 ans. La Mousse est le nom d'un fief situé à Saussay, près de Saint-Remy. 
François Jean-Charles de Croisilles s'est éteinte dans les personnes de JeanClaude de Croisilles, seigneur de Bretteville-sur-Bordel, et de Dominique de Croisilles, sieur de Putot ${ }^{32}$.

Hormis ces quelques collatéraux éloignés, les proches et les domestiques accusés de s'être emparés de l'esprit de la dame de Croisilles, et le fils indigne coupable de l'avoir enfermée, les courriers n'évoquent que la figure du comte de Blangy. Marie-Madeleine de Méhérenc explique qu'on a relaté à ce dernier des faits imaginaires, qu'il a été en conséquence désinformé et manipulé. Le seigneur de Villers-Bocage a été dans cette affaire un acteur d'importance, puisqu'il est l'auteur de quatre courriers écrits à un moment crucial, entre l'envoi du placet au ministre intervenu à la date du 10 juillet et les ordres d'arrestation adressés par Bertin à son intendant le 16 août. Blangy déconseille d'abord fortement au fils d'entreprendre un procès, au motif qu'il n'a pas les moyens financiers de le soutenir et parce que l'issue lui paraît trop incertaine. L'incarcération de la mère par lettre de cachet est également délicate. "Elle est sans exemple... Rien ne me paraît plus difficile ». Toutefois, il ne voit guère d'autre action possible! La dissipation de l'héritage maternel surtout le préoccupe. À deux reprises il expose sa crainte de voir la dame de Croisilles instruite des démarches du fils tout vendre moitié au-dessous de sa valeur. Blangy correspond directement avec le ministre et demande à un interlocuteur, qui ne peut être que l'intendant de Caen, Charles Esmangart, de certifier au ministre la vérité de son exposé. C'est le 16 août 1778, à la suite de ces échanges, que le ministre Bertin donne des ordres à Esmangart pour qu'il soit procédé à l'arrestation de la mère. Ce n'est donc pas sans raison que les auteurs d'un mémoire anonyme en défense de la dame font état du caractère arbitraire de la procédure : "Il est question d'une cause commune à tous les pères de famille qui doivent trembler sur leur existence si leurs enfants, fâchés d'en voir prolonger le cours, ont quelques amis puissants ${ }^{33}$. " Blangy n'apparaissant plus par la suite, son rôle - crucial en réalité - s'est limité à convaincre le ministre d'envoyer les ordres d'enfermement.

Une fois obtenue l'incarcération, le rôle principal revient au curateur nommé à la dame, c'est-à-dire à Cauvin de Beaumont. On n'accusera pas le curateur d'être pécuniairement intéressé au maintien de la dame au couvent, puisque personne parmi les défenseurs de celle-ci ne lance une telle accusation. Mais un mémoire dit tout de même que le fils et ses amis ont exercé " une odieuse inquisition dans les meubles et papiers de sa mère ". Il est ajouté que de l'argent et des papiers pourraient avoir été soustraits et qu'une vendue (vente) des meubles semble avoir été envisagée. Cette

32. Jean-Claude de Croisilles, écuyer, seigneur et patron de Bretteville, du Mesnil-Vité, de Saint-Martin-de Fresnay, de Montviette et d'Ecots, né le 12 janvier 1654, fils aîné de Robert de Croisilles, conseiller au présidial de Caen, et d'Anne Cairon, fut installé président au présidial en mai 1704; il fut directeur de l'Académie de Caen en 1705 et en 1712, et mourut sans postérité, le 21 janvier 1735 .

33. Arch. dép. du Calvados, C 337, mémoire anonyme non daté (automne 1778) adressé à l'intendant. 
dernière ne pouvant être décidée que par le curateur, il est sûr que Cauvin de Beaumont figurait parmi les " amis " précédemment évoqués. Cauvin, qui se déclare porte-parole de toute la famille, désapprouve le fils, lorsque celui-ci renonce à la contrainte et demande à faire libérer sa mère. Il écrit même au ministre pour l'en dissuader. Une lettre, rédigée à l'intention de Bertin, s'étend longuement sur les manœuvres du camp adverse ${ }^{34}$. Cauvin revient d'abord sur une lettre écrite le 5 octobre 1778 par les " parents" de la dame de Croisilles à l'intention de l'intendant pour obtenir sa libération : "La plupart des parents ayant signé pour Madame de Croisilles étaient postiches ", l'un d'eux n'ayant que 15 ans. "Le surplus sont gens comme il faut, mais point parents et ne connaissant pas même la dame de Croisilles ". Deux hommes sont spécialement visés par Cauvin, deux avocats entrés au service de la dame. Desmares est accusé d'avoir écrit un plaidoyer " très déplacé " contre le comte de Blangy et d'avoir détourné la dame de Croisilles de tous les arrangements honnêtes qu'il lui avait proposés. La lettre, écrite le $1^{\mathrm{er}}$ mars 1779 , intervient après que le fils a envoyé un nouveau placet en janvier en vue d'obtenir la libération de sa mère. Elle cherche à empêcher celle-ci.

Mieux valait, pour obtenir une incarcération aussi scandaleuse - puisqu'il s'agit d'une personne majeure et maîtresse d'elle-même - le soutien de personnalités influentes. La dame bénéficia du changement d'état d'esprit de la seconde moitié du XVIII ${ }^{\mathrm{e}}$ siècle, et de la réticence des autorités à s'entremettre dans les affaires privées, conjugales ou filiales. L'État n'entendait plus se mêler de ces sortes d'affaires si n'y étaient pas mêlés un désordre et un scandale publics.

\section{Sentiments lignagers et liberté féminine}

L'enchaînement des faits, tels qu'ils nous sont relatés par les différents acteurs, donne à penser que l'accusation de dissipation n'était pas tout à fait injustifiée. Cependant, elle ne semble pas avoir été à l'origine de tout. On peut s'en convaincre en évaluant les forces en présence, l'origine des biens et le pouvoir de chacun. On peut s'en convaincre surtout en examinant les mobiles de la dame de Croisilles. Un document daté de 1749, postérieur à la séparation de biens, indique que le mari devait 19500 livres de remplacements pour avoir aliéné des biens appartenant à son épouse ${ }^{35}$. On ne sait quelle était la provenance de ces biens. Exilée en Bretagne, la dame de Croisilles ne reçut de l'héritage de son père qu'une modeste pension de 800 livres, négociée par son mari, haussée à 1000 livres après la mort de sa mère. Lors de son enfermement à l'abbaye de la Blanche de Mortain, la pension fixée cette fois à 1200 livres ne représentait toujours qu'une modeste portion des revenus de la dame puisque le revenu de la terre de Cardonville valait 7000 livres. Le mari de la dame conserva la jouissance du

34. Arch. dép. du Calvados, C 337, lettre de Cauvin de Beaumont du $1^{\text {er }}$ mars 1779.

35. Arch. dép. du Calvados, F 1073. 
reste jusqu'à son décès. Et la dame de Croisilles se retrouva en possession du tout en 1770. Le fils voulut-il empêcher la perte des héritages maternels? La vente d'un herbage valant 15000 livres, faite le 6 mars 1778, semble avoir été le déclencheur de toute la suite. L'inquiétude était-elle si grande, la menace si urgente et si précise, qu'il ait fallu procéder dans la précipitation et dans le secret à une action aussi choquante que l'internement d'une mère obtenu par son fils!

Pour comprendre ce qui se joue, il faut revenir au début de l'histoire et au mariage de Bernardin de Croisilles avec la dame de Méhérenc. En 1778, l'évènement paraît si éloigné que la veuve Croisilles, âgée de 66 ans, ne sait plus le dater : " J'épousai il y a 40 à 45 ans Bernardin de Croisilles, escuier. Sa fortune n'était pas égale à celle que je devais posséder un jour. » Le bien du mari valait 3000 livres quand le sien, disait-elle, dépassait 5000 livres $^{36}$. Ce mariage, dissymétrique sans être disproportionné, tourna rapidement si mal que la femme fut enfermée une première fois par lettre de cachet en 1747. Lorsque Marie-Madeleine de Méhérenc fut internée en 1778, il s'agissait donc d'un second enfermement, le fils prenant en cela la voie déjà suivie par le mari. Les premières difficultés naquirent du commerce indécent entretenu par l'épouse avec un commis aux aides que le mari parvint à écarter de la ville. C'est volontairement qu'elle accepta de se retirer à l'hôtel-Dieu de Caen. La dame avait aussi quelques reproches à faire à son mari. Ce dernier était, disait-elle, dans une telle connivence avec ses propres parents qu'elle ne le supportait plus : "Comme il est fort attaché à son père et à sa mère, il a toujours voulu demeurer avec eux. Ce que j'ai patienté pendant nombre d'années, mais à la fin, ne pouvant plus y tenir, par les conseils que je m'apercevais qu'il [son beau-père] donoist à mon mari, j'ai pris le parti de me retirer à l'hôtel-Dieu ${ }^{37}$. " À Caen, la dame de Croisilles se retrouva à proximité de sa famille. Si son mari la menaça alors d'employer contre elle une lettre de cachet, ce fut, dit-elle, pour l'éloigner de sa famille et la forcer à revenir auprès de lui à Villers-Bocage. Mais elle refusait " de retourner" dans la maison des Croisilles. La suite est connue. Elle fut mise à l'abbaye Blanche des bénédictines de Domfront, puis transférée en la communauté de Montbareil à Guingamp. Après trois années, en octobre 1751 , son mari consentit à la faire sortir ${ }^{38}$. De là, elle gagna Rennes et demeura plusieurs années chez un perruquier où elle se prit d'affection pour l'enfant dont il a été plusieurs fois question.

L'adultère alors, plutôt que la dissipation, serait-il à l'origine de tout? L'éloignement de l'amant ne permit pas de faire revenir la paix dans le ménage. Celui de l'épouse ne convint pas non plus, car il nuisait tout autant

36. Arch. dép. du Calvados, C 337, lettre de la dame de Croisilles à Bertin, 11 septembre 1778 .

37. Arch. dép. du Calvados, C 337, mémoire de la dame de Croisilles à La Briffe, intendant de Caen, 10 novembre 1747.

38. Arch. dép. du Calvados, C 337, lettre de Bernardin de Croisilles à $\mathrm{M}^{\mathrm{gr}}$ le comte de Saint-Florentin, ministre, 13 octobre 1751. 
à la réputation du mari. Il est probable que Bernardin de Croisilles a cherché à obtenir son retour auprès d'elle, non point par sentiment, mais pour la préservation de son honneur. C'est bien elle qui s'y refusa obstinément, comme plus tard, quand enfermée une seconde fois, elle s'obstina à ne rien signer. La préservation de l'honneur du mari exigeait qu'elle fût tenue à distance de son fait à lui, après une décision judiciaire ou administrative, et non point par la volonté de son épouse! La première lettre de cachet fut prise, non pas pour préserver un secret, mais au contraire pour être connue et laver un affront. Après trois années, par clémence et parce que son éloignement ne risquait plus cette fois de faire jaser, il consentit à ce qu'elle sortît. L'extraordinaire de l'affaire est l'entêtement de l'épouse à préférer le couvent à sa belle-famille! Même l'éloignement de son fils n'y fit rien. Une femme, capable de sentiments maternels, comme la suite semble le démontrer, trouve la force de rompre avec mari, fils et conventions au point d'encourir un enfermement et un exil. Le motif en est qu'elle ne veut pas " retourner "! L'écart d'âge entre les deux époux - 34 ans - fournit la clef de toute l'affaire. Il s'est agi d'un mariage arrangé, par conséquent d'une combinaison matrimoniale n'ayant eu en vue que les intérêts du patrilignage.

"Point de signature, ne m'en voulez point de $\mathrm{mal}^{39}$ ! " Cette rupture avec les conventions sociales du temps et du lieu est délibérée. La dame accueille chez elle des soldats, achète un carrosse pour les promenades de sa gouvernante-dame de compagnie, exprime sans réserve toute l'estime qu'elle a pour un perruquier, fait venir un maître pour le fils de celui-ci, déclare que sa servante a une noblesse de sentiments bien préférable à celle qu'on reçoit de ses aïeux. Si elle montre une pointe de mépris au sujet du mariage contracté par son fils avec la fille d'un Canadien, aucun propos ne donne à penser qu'elle s'offusque d'une alliance socialement médiocre. François Jean-Charles de Croisilles, son fils légitime, lieutenant au régiment de la Couronne, a épousé Marie-Catherine Héry le 6 janvier 1770 à l'âge de 29 ans. Elle est la fille d'un négociant canadien, Charles Héry, qui a quitté la colonie depuis plusieurs années. Charles Héry procéda en effet à la vente de ses propriétés canadiennes au cours de l'été 1764 et au début de l'automne 1765 , avant de gagner la France. Le père était assez riche pour que les filles puissent espérer des alliances dans la petite noblesse métropolitaine ${ }^{40}$. Mais Charles Héry était le fils d'un Jacques Duplanty-Héry, bourgeois de Montréal, lui-même fils d'un marchand tanneur de Saint-Jean-d'Angély. Les Croisilles n'étaient clairement pas sur une pente ascendante! Cela pourtant, observons-le, laissa la dame de Croisilles tout à fait indifférente. Elle n'avait plus d'yeux que pour son enfant adoptif - un enfant naturel de la dame? (!) - qui avait grandi auprès d'elle et dont elle s'était occupé, tandis qu'elle n'avait plus vu son fils légitime depuis deux décennies. À une femme

39. Arch. dép. du Calvados, C 337, lettre de la dame de Croisilles, 2 décembre 1778.

40. Agathe Héry épouse en 1772 Pierre Jacques Joseph Mullon de la Rochellière, seigneur d'Eytré. 
de la petite noblesse normande, livrée aux intérêts patrilignagers, il n'est guère en définitive d'autre ressource que la résistance passive.

Le rejet du fils à la suite de celui du père s'explique très bien en contexte patrilignager. Les mâles en Normandie faisaient ligne, et cela suffisait à mettre la femme dans une position subordonnée. Marie-Madeleine de Méhérenc, mariée par intérêt à un homme beaucoup plus âgé qu'elle, a rapidement défié l'ordre patrilignager. C'est le caractère public de la rébellion, tournée contre ce pilier de l'ordre social et familial normand qu'est le patrilignage, c'est ensuite la solidarité de classe et de genre manifestée au niveau local par les élites locales, qui aboutit à une mesure aussi grave et exceptionnelle que l'internement d'une veuve et d'une mère. La lettre de cachet, qui n'était pas ordinairement accordée pour des dérangements bénins, restaura ici l'honneur des mâles. Mais ce qu'un mari avait obtenu contre une épouse indocile et infidèle, un fils ne pouvait plus l'obtenir contre sa mère, trente ans plus tard. Le principe selon lequel la femme, en dehors des liens du mariage, demeurait maîtresse de son patrimoine, était universellement reçu, et désormais brandi à Versailles contre les demandes abusives. Il fallait être normand, et avoir le soutien du principal seigneur du lieu, pour oser requérir une telle mesure d'enfermement, même provisoire. En ce sens, l'affaire ici exposée témoigne simultanément d'un phénomène général - la crise du couple et la remise en question de l'autorité maritale et d'un phénomène spécifiquement normand, qui est la persistance dans cette province d'un esprit patrilignager ignoré ailleurs à un tel degré. 


\section{RÉSUMÉ}

L'affaire dont il est question se déroule en deux temps. Marie Madeleine de Meherenc, dame de Croisille, est enfermée à l'hôtel Dieu de Caen en 1747. Elle y séjourne de son plein gré, peut-être déjà sous la menace d'une lettre de cachet. Son mari a en effet à lui reprocher une inconduite et même un commerce scandaleux avec un commis aux aides. Mécontent de constater que son épouse trouve encore à circuler librement, il obtient des ordres du roi pour la faire enfermer en un lieu plus sérieux. C'est le début d'une errance, qui conduit la Dame à Domfront puis à Guingamp. On ne sait presque rien des 23 années suivantes. À la mort de son mari, survenue en 1770, elle réapparaît à Villers-Bocage. Elle y cohabite un temps avec son fils, François Jean Charles de Croisilles et sa bru, avant de les expulser de sa maison. La suite des évènements convainc le public qu'elle déteste son fils, qu'elle veut le priver de tous ses biens. En 1778, tandis qu'elle a 66 ans, son fils la fait à nouveau enfermer. L'affaire, richement documentée, sur un arrière-plan patrimonial, mêle affects, sentiments conjugaux et filiaux. L'affaire ici exposée témoigne simultanément d'un phénomène général - la crise du couple et la remise en question de l'autorité maritale à la fin de l'Ancien Régime - et d'un phénomène plus spécifiquement normand, qui est la persistance dans cette province d'un esprit patrilignager ignoré ailleurs à un tel degré.

\section{ABSTRACT}

In 1747 Marie Madeleine de Meherenc, lady of Croisille, was shut away in the hôtel-Dieu of Caen. She stayed there of her own will, perhaps under the threat of a lettre de cachet. Her husband had reproached her ill-conduct and even scandalous commerce with a financial clerk. Disappointed that his wife was still able to move freely, he obtained royal letters to have her locked up in a more secure place. This was the starting point for a series of moves that led Meherenc to Domfront then Guingamp. We know little of the following twenty-three years. At the death of her husband in 1770 she reappeared in Villers-Bocage. She stayed there for a while with her son, François Jean Charles de Croisilles and her daughter-in-law, before expelling them. Her acts thereafter convinced the wider public that she hated her son and that she sought to deprive him of her inheritance. In 1778, when she was aged 66, her son had her once more shut away. This is a well-documented affair of inheritance and complex feelings, of marital and filial affection. The affair analysed here bears witness to a general phenomenon - marital crisis and the question of authority at the end of the Ancien Régime - and a more specifically Norman phenomenon, that is the persistence in this province of a patrilineal approach that has hitherto been overlooked. 